\title{
A Cognitive Radio-Based Energy-Efficient System for Power Transmission Line Monitoring in Smart Grids
}

\author{
Saeed Ahmed, Young Doo Lee, Seung Ho Hyun, and Insoo Koo \\ School of Electrical Engineering, University of Ulsan, 93 Daehok-ro, Nam-gu, Ulsan 44610, Republic of Korea \\ Correspondence should be addressed to Seung Ho Hyun; takeitez@ulsan.ac.kr
}

Received 27 June 2017; Revised 8 November 2017; Accepted 14 November 2017; Published 19 December 2017

Academic Editor: Guiyun Tian

Copyright ( 2017 Saeed Ahmed et al. This is an open access article distributed under the Creative Commons Attribution License, which permits unrestricted use, distribution, and reproduction in any medium, provided the original work is properly cited.

\begin{abstract}
The research in industry and academia on smart grids is predominantly focused on the regulation of generated power and management of its consumption. Because transmission of bulk-generated power to the consumer is immensely reliant on secure and efficient transmission grids, comprising huge electrical and mechanical assets spanning a vast geographic area, there is an impending need to focus on the transmission grids as well. Despite the challenges in wireless technologies for SGs, cognitive radio networks are considered promising for provisioning of communications services to SGs. In this paper, first, we present an IEEE 802.22 wireless regional area network cognitive radio-based network model for smart monitoring of transmission lines. Then, for a prolonged lifetime of battery finite monitoring network, we formulate the spectrum resource allocation problem as an energy efficiency maximization problem, which is a nonlinear integer programming problem. To solve this problem in an easier way, we propose an energy-efficient resource-assignment scheme based on the Hungarian method. Performance analysis shows that, compared to a pure opportunistic assignment scheme with a throughput maximization objective and compared to a random scheme, the proposed scheme results in an enhanced lifetime while consuming less battery energy without compromising throughput performance.
\end{abstract}

\section{Introduction}

The concept of the smart grid (SG) is to insert adequate intelligence to augment control of the traditional electric grid and make it more autonomous. Application-oriented communications technology, supported by advanced sensors and actuators, constructs the foundation of smart procedures to generate, transmit, and distribute power to consumers, enabling them to understand and control electricity consumption.

Substantial research is being carried out in communications technologies for the SG. However, most of the investigation by academia and industry has primarily focused on the distribution grid and consumption-side management, leaving the vast picture of the transmission grid uncertain. Huge electrical and mechanical assets of transmission grids, spanning thousands of kilometers, are highly exposed to numerous forms of hostile, natural, and artificial destruction, compromising the overall stability, security, and performance of the electric power grid. Therefore, in the context of the SG, an appropriate two-way communications network is imminently needed for real-time structural awareness, fault identification, localization, and elimination by the power control center (PCC), guaranteeing reductions in maintenance cost and enhancing the stability and security of the power supply system. Wireless sensors are put in various components of transmission lines to collect different physical and electrical parameters of the transmission lines (like sagging, vibration, electric current density, icing effects, vegetation, overheating, etc.) $[1,2]$. However, delivering the huge amount of collected information to the PCC, located miles away, in a long hop-by-hop linear topology is the most critical task in developing a smart transmission grid (STG) due to short ranges, low data rates, less successful data delivery, unbalanced energy consumption, and higher data collisions in wireless sensors. Hybrid network-based solutions deploying the transmission tower-mounted sensors, to collect the parameters and the cellular Global System for Mobile Communications (GSM), to deliver the collected data to the PCC have been proposed in the literature for 
power transmission line monitoring. Battery-limited wireless devices employed for data delivery in the monitoring network are required to keep functioning uninterruptedly for a long period, and frequent battery replacement for these devices is not feasible. Thus, limited battery energy may become a barrier to the design of power transmission line monitoring networks. In this context, higher energy efficiency is sought in the design of the monitoring network for an elongated lifetime, cost-effectiveness, and global warming concerns [3]. From the STG management viewpoint, energy efficiency means monitoring the transmission grid for long durations. Prior efforts in power transmission line monitoring not only incur huge cost and face service coverage issues but also do not consider the efficient utilization of limited battery energy of the wireless nodes in their design criteria.

Contrary to prior proposed technologies, we propose an energy-efficient two-level power transmission line monitoring system, based on cognitive radio IEEE 802.22 wireless regional area network (WRAN). The CR IEEE 802.22 is potentially considered a highly attractive technology to satisfy the communications needs of smart transmission grids (STGs) [4]. To the best of our knowledge, this paper is the first CR-based work in power transmission line monitoring aiming at improvement in energy efficiency on channel assignment, resulting in an extended lifetime of the monitoring network. Contributions of our paper can be summarized as follows:

(i) Based on the requirements of long lifetime and less time delay for a power transmission line monitoring network, we propose a cluster-divided twolevel CR 802.22 WRAN-based power transmission line monitoring system, in which first, the electrical and mechanical parameters of a transmission line in a cluster are collected and forwarded by infrastructure-mounted local sensors to cognitive relays attached in certain locations on power line transmission poles (termed cognitive poles in the rest of this paper). Collected data are then delivered by the cognitive poles to the PCC via a central entity in each cluster, that is, a cognitive radio gateway (CRGW).

(ii) Considering the battery limitations of the cognitive poles, we formulate the channel assignment problem at the CRGW as an energy efficiency maximization problem which is a nonlinear programming (NLP) problem. To overcome the computational intricacies, we propose an energy-efficient channel assignment scheme (ECAS) as a solution to this problem. Our proposed scheme solves the problem through the Hungarian method to find optimal energy efficiency for a cluster thus resulting in extended monitoring network lifetime without compromising the throughput performance of the network.

(iii) Numerical analysis shows that proposed ECAS reduces the overall energy consumption in a cluster by assigning the appropriate channels to the cognitive poles without compromising throughput performance and is capable of elongated monitoring.

The rest of this paper is organized as follows. In Section 2, we review prior efforts at transmission line monitoring in the context of the SG and discuss the issues related to the earlier work. Section 3 introduces the CR-based system model for transmission line monitoring. Section 4 presents the problem formulation, along with the proposed solution. Performance evaluation is carried out in Section 5 to show the effectiveness of our proposed scheme. Finally, we conclude this paper in Section 6 and describe the directions of our future work.

\section{Related Work}

Dedicated wired broadband and power line-dependent communications methods implemented for indoor environment [5] are impractical to implement a grid-wide monitoring system because they need huge lengths of expensive cables. If any of the supports fail or if power lines break, the monitoring data delivery is halted, and fault identification becomes enormously problematic. Aside from the wired approaches, putting wireless sensors in acute locations in the path of the transmission line to monitor the fine-grained status of different electrical and mechanical parameters has been proposed $[6,7]$. Wireless sensor nodes are considered an integral part of the smart transmission grid (STG) due to the features like low cost, easy installation, and rational coverage areas [8, 9]. However, the authors did not specify the underlying network for the critical task of dispatching the huge amount of collected data to the PCC, located miles from the transmission lines. Len et al. [10] and Chen et al. [11] were the first to propose a two-level wireless sensor-based model to observe and transmit data to the PCC in a hop-by-hop relay manner. Transmission poles are roughly deployed in a line; thus, the sensor network is linear in topology. Lin et al. [12] proposed a dense set-based clustering algorithm upon hybrid media access control by rotating the role of relaying node to create an intracluster energy balance considering the linear topology. Taking into consideration linear topology limitations, with low data-carrying capacity and a short range for communication, a pure wireless sensor node-based network would fail to successfully deliver the data to the PCC. In a linear sensor network, the effective delivery ratio of data in the nodes farther from the substation is found to be much smaller than for nodes closer to the substation because data from a farther node essentially travels over a longer distance, and the collision rate is higher. In addition, the workload is not balanced for the relaying sensors, because the sensors near the substations must deliver more data, exhausting their battery energy rapidly, compared to other sensors [13]. Considering the bottlenecks associated with a linear chain sensor node-based monitoring system, Hung et al. [1] proposed a hierarchal model considering a combination of cellular transceivers mounted on each transmission tower, along with wireless sensors to collect and transmit the power transmission line status. Huge installation and subscription costs involved in this work inspired the researchers to find optimal 
TABLE 1: A comparison of the notable features of existing and proposed system models.

\begin{tabular}{|c|c|c|c|}
\hline $\begin{array}{l}\text { Communications technology } \\
\text { Features }\end{array}$ & $\begin{array}{l}\text { Wireless sensor nodes } \\
\text { (ZigBee, Bluetooth) }\end{array}$ & $\begin{array}{l}\text { Hybrid network } \\
\text { (WSN + cellular) }\end{array}$ & $\begin{array}{l}\text { CR } 802.22 \text { WRAN } \\
\text { (proposed) }\end{array}$ \\
\hline Network topology & Linear & Linear & Star (clustered) \\
\hline Energy efficiency considered in design & $\mathrm{x}$ & $\mathrm{x}$ & $\checkmark$ \\
\hline Overall network energy efficiency & Low (unbalance workload) & Low (unbalance workload) & High (balanced workload) \\
\hline Network lifetime & Low & Low & High \\
\hline Data rate & Low & High & High \\
\hline Communication range & Low (few hundred meters) & Service coverage dependent & $\operatorname{High}(33-100 \mathrm{~km})$ \\
\hline Spectrum type & Unlicensed & Licensed & Unlicensed \\
\hline Spectrum cost & Free & High & Free \\
\hline Uncontrolled interference & $\checkmark$ & $\checkmark$ & $\mathrm{x}$ \\
\hline Wideband spectrum & $\mathrm{x}$ & $\mathrm{x}$ & $\checkmark$ \\
\hline Time delay & High & Moderate & Low \\
\hline Power adaptation & $\mathrm{x}$ & $\mathrm{x}$ & $\checkmark$ \\
\hline References & {$[3-12]$} & [13-15] & {$[4,17]$} \\
\hline
\end{tabular}

locations for cellular transceivers along the length of the transmission line. Wu et al. [14] and Fateh et al. [15] proposed a hierarchical model to find the optimal poles equipped with the cellular capability to reduce the installation costs. These solutions experienced huge installation, maintenance, and spectrum subscription costs. The availability of cellular service along the complete path of the transmission line and service outage issues are additional hampering factors in the deployment of the cellular-based power transmission line monitoring. The data relay nodes are limited battery devices. Existing proposals did not investigate how to enhance the lifetime of the battery limited monitoring system. For elongated monitoring of the transmission grid assets, the power supply to the data relay nodes is critically important.

Huertas et al. [16] evaluated the effects of EM interference on wireless data transmission from inside the EM fields of transmission lines and concluded that the presence of EM interference by high-voltage transmission lines can reduce wireless data transmission. Therefore, powering the cognitive poles through magnetic or capacitive induction of highvoltage lines requires complex electronic circuitry, along with costly insulation kits, to mitigate the effects of large electromagnetic interference, increasing the overall cost of gridwide deployment of a monitoring system [18]. Similarly, horizontal axis wind turbines and solar panels are commonly not considered suitable power supply sources for transmission line monitoring sensor nodes, because they need to be fitted on the transmission towers, which incurs huge installation and maintenance costs [19]. Thus, battery limitations of the relay nodes become a hampering factor in the design of monitoring system for power transmission lines. Generally, high-voltage transmission lines run through less populated remote areas because most of the power-producing plants are constructed away from cities. Because the power transmission line monitoring is specific in nature, it is necessary to develop a monitoring system based on the communications technology which considers the prolonged lifetime and cost-effectiveness.
Contrary to the license-based communications technologies, the IEEE 802.22 standard developed for WRANs employing TV white space (TVWS) with CR technologies can provide effective subscription cost-free utilization of idle frequency spectrum with a reasonably good coverage area [20]. An 802.22 CR WRAN, which was proposed for providing broadband internet services to remote rural areas in [21], can have numerous attractive features for SG applications. In [22], Brew et al. proposed the network architecture and investigated the role of $\mathrm{CR}$ communications in rural areas to support distributed generation of electricity. In [23], Li et al. proposed a CR-based power transmission line monitoring model to address the issue at the very high level of abstraction without specifying the underlying network required for delivery of data to PCC. Majority of the existing works, however, did not consider the battery energy constraint for an elongated lifetime in the context of the power transmission line monitoring network. In this paper, we exploit the attractive features of the CR 802.22 WRAN technology in order to propose a novel energy-efficient power transmission line monitoring system. Table 1 illustrates the notable features of our proposed network in comparison to the existing models.

\section{System Model}

Figure 1 illustrates the proposed architecture of a two-level smart transmission grid consisting of an overhead highvoltage transmission line running between two substations. Substations are linked to the PCC through optical fiber cable. A typical distance between two substations is about $50 \mathrm{~km}$. The transmission line is supported by poles, and the distance between two consecutive poles can be between 0.5 and $1 \mathrm{~km}$, depending upon the terrain.

At level 1, infrastructure-mounted local sensors measure different parameters, such as conductor temperature, sagging, wind pressure, vibration, and current intensity, and forward their observations to cognitive poles. At level 2, cognitive poles collect data from local sensors to form packets 


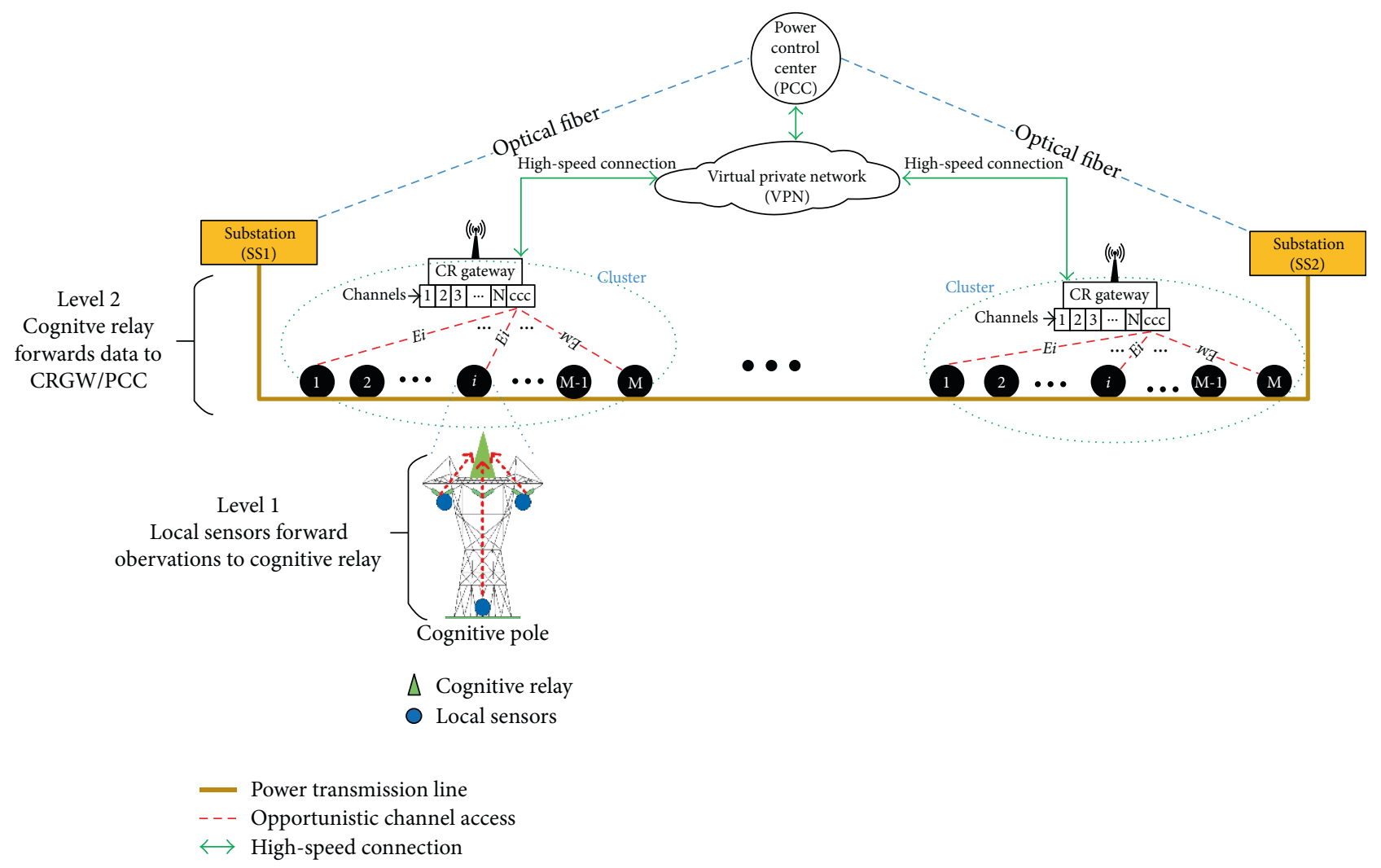

Figure 1: Two-level smart transmission grid system model.

of fixed size and report their observations to the PCC after getting the CAM from the CRGW. The CRGW is responsible for allocating channels to cognitive poles, and it is also connected to the PCC through a high-speed data link. Thus, there can be 50 to 100 poles between two substations.

We consider a clustered CRN architecture serving $M$ cognitive poles, $(i=1,2, \ldots, M)$, where a central entity CRGW acts as a cluster head and cognitive poles are the members of the cluster. The distance of the cognitive poles from the CRGW is the criteria for the formulation of clusters. The primary network (PN) has $N$ nonoverlapping orthogonal channels. The occupancy state of each channel is modeled as a two-state Markov chain. The channel that is idle has the probability $p_{\text {idle }}$. It is assumed that primary user (PU) spectrum occupancy is obtained by the CRGW from a reliable external entity, such as a white space database [24], and spectrum sensing is not performed. We assume that the CRGW database is completely synchronized with the $\mathrm{PN}$ and the CRGW is responsible for allocating $j$ idle channels to $M$ cognitive poles in a cluster, where $j=1,2, \ldots, N$.

Considering the frame architecture shown in Figure 2, in a cluster at the beginning of each frame, the CRGW broadcasts the idle channel information to all cognitive poles on a common control channel. In response, each pole sends a small packet on different idle channels to the CRGW. Each pole also shares its residual battery energy level, $E_{i}$, with a cluster gateway on the common control channel. Exchange of this information is completed in the control message duration, $T_{\mathrm{ctr}}$, for each frame. We assume that control message duration is significantly shorter, compared to other periods of the frame. As all the information is gathered at the CRGW, the CRGW executes an assignment policy and broadcasts the information to all the cognitive poles. After that, a pole can be either in transmission mode or idle mode. Figure 2 illustrates both modes.

3.1. Mathematical Modeling for Channel Capacity. The maximum number of bits that cognitive pole $i$ can send through a channel $j$ in a frame depends on the following three factors: bandwidth of the channel $W$, SNR of the channel $j$ for cognitive pole $i$, and channel switching delay, Tcs. The channel switching delay is the time that is spent in tuning the cognitive pole's radio frequency front-end from the previous channel to the newly assigned channel. It also serves as a key factor in determining the total number of bits that a cognitive pole can send on the assigned channel. In the literature, this is termed channel switching latency. It is reflected as a linear function of the total channel distance between the previous $j^{\prime}$ and the current channels $j$ [25-28]. Therefore, channel switching latency denoted by $T_{\mathrm{cs}}$ is given as follows:

$$
T_{\mathrm{cs}}=\tau_{\mathrm{cs}}\left|\left(j^{\prime}-j\right)\right| \text { seconds, }
$$

where $\tau_{\text {cs }}$ is the switching unit bandwidth delay. Let $C_{i, j}$ be the capacity of the channel $j$ for cognitive pole $i$. Using the Shannon-Hartley equation, we calculate it as follows: 


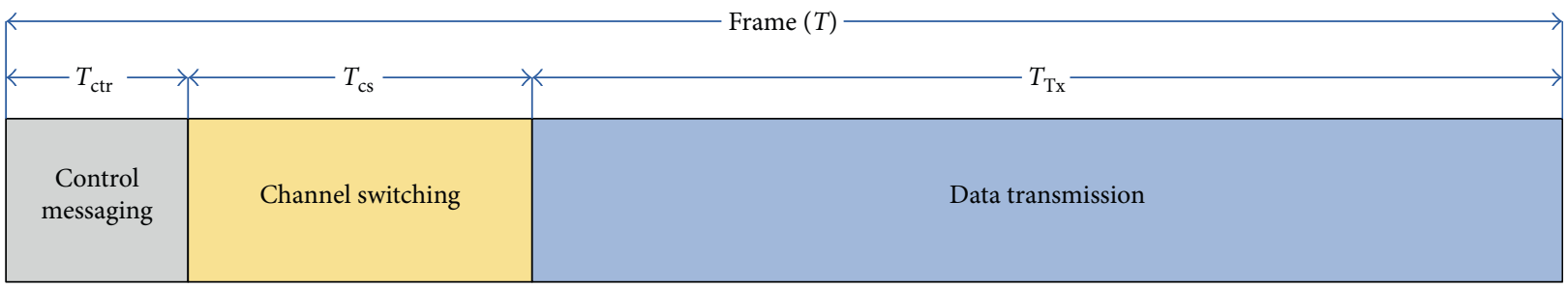

(a)

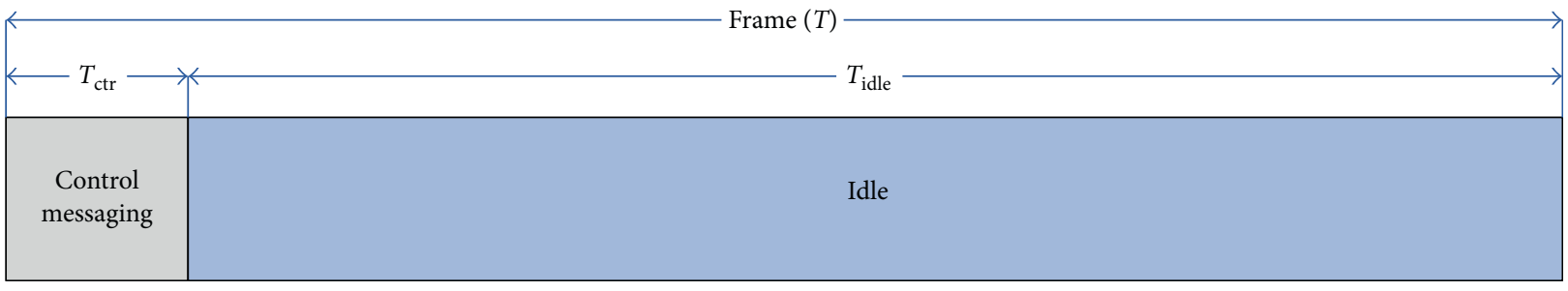

(b)

FIGURE 2: Frame organization of the proposed energy-efficient channel allocation scheme: (a) frame structure of cognitive poles for control messaging, channel switching, and data transmission when the channels are assigned by the CRGW; (b) frame structure of cognitive poles for control messaging and idling duration when the channels are not assigned by the CRGW.

$$
C_{i, j}=W \log _{2}\left(1+P_{\max }^{\mathrm{Tx}} \frac{\left|h_{i, j}\right|}{N_{0}}\right) \text { bits/second, }
$$

where $\left|h_{i, j}\right|, N_{0}$, and $P_{\max }^{\mathrm{Tx}}$ are the gain of the channel, the normalized noise power, and the maximum transmission power, respectively. The actual throughput of a channel is the maximum number of bits that can be sent by a cognitive pole on the channel during a frame. Let $M_{i, j}$ denote the actual throughput of the channel $j$ when cognitive pole $i$ transmits on this channel. It is calculated as follows:

$$
M_{i, j}=C_{i, j}\left(T-T_{\mathrm{ctr}}-T_{\mathrm{cs}}^{i, j}\right) \text { bits, }
$$

where $T, T_{\mathrm{ctr}}$, and $T_{\mathrm{cs}}^{i, j}$ are the frame duration, the control message duration when the cognitive pole $i$ is in transmission mode, and the time consumed by a cognitive pole $i$ in switching the channel when the assigned channel $j$, respectively. Therefore, the total throughput of the cognitive pole network can be calculated as follows:

$$
R=\sum_{i=1}^{M} \sum_{j=1}^{N} D_{i, j} M_{i, j} \text { bits, }
$$

where $D_{i, j}$ is the binary decision variable that represents the assignment status for cognitive pole on the channel $j$. If the value of $D_{i, j}=1$, then the CRGW assigns a channel to cognitive pole $i$; otherwise, the value of $D_{i, j}=0$. Hence, and there will be no assignment of a channel to the cognitive pole.

\subsection{Mathematical Modeling for Energy Consumption. Let $\mathbb{S}$} denote the set of cognitive poles that have been assigned channels by the CRGW. The frame structure in Figure 2(a) shows that if a cognitive pole is assigned a channel (i.e., $i \in \mathbb{S}$ ) by the CRGW, it switches its hardware to the assigned channel, taking $T_{\text {cs }}$ units of time, and transmits till the end of the frame. We refer to this scenario as the transmission mode scenario. On the other hand, Figure 2(b) illustrates that if a pole is not assigned a channel (i.e., $i \notin \mathbb{S}$ ), it stays idle after the control message time till the end of the frame. We refer to this scenario as the idle mode scenario. Accordingly, we model the energy consumption for both scenarios as follows.

\subsubsection{Energy Consumption in Transmission Mode Scenario.} We calculate the energy consumption by a cognitive pole during different stages of transmission in this subsection. At the beginning of a frame, each cognitive pole sends its state to the CRGW in the $T_{\text {ctr }}$ time duration, consuming control messaging energy $E_{\mathrm{ctr}}^{\mathrm{Tx}}$. The energy consumed by the pole $i$ during a control messaging task is calculated as follows:

$$
E_{\mathrm{ctr}}^{\mathrm{Tx}}=P_{\max }^{\mathrm{Tx}} T_{\text {ctr }} \text { joules. }
$$

We assume that the cognitive poles use maximum transmission power $P_{\max }^{\mathrm{Tx}}$ during the control message duration. After the control message, the poles that are assigned different idle channels by the CRGW switch to the assigned channels by consuming channel switching energy $E^{c s}$. Channel switching energy consumption by pole $i$ when assigned channel $j$ is calculated as follows:

$$
E^{\mathrm{cs}}=P^{\mathrm{cs}} T_{\mathrm{cs}}^{i, j}
$$

where $P^{c s}$ is the power dissipation by a cognitive pole for channel switching. The channel switching duration from the previous channel $j^{\prime}$ to the new channel $j$ is given as $T_{\text {cs }}^{i, j}=\tau_{c s}\left|\left(j^{\prime}-j\right)\right|$. After channel switching and control messaging, transmission time decreases to $\left(T-T_{\text {ctr }}^{\mathrm{Tx}}-T_{\mathrm{cs}}\right)$ seconds. Finally, the cognitive pole starts transmitting the collected power line parameters on the assigned channels, consuming energy $E_{\text {data }}^{\mathrm{Tx}}$. In this paper, we have considered adaptive transmission power control in which the transmission power during data transmission is adaptively assigned (up to the maximum allowable transmission power 
range) to the cognitive pole according to the assigned channel condition.

Transmission energy consumption is proportional to the transmission duration and the assigned transmission power $P^{\mathrm{Tx}}$. The transmission duration $\tau_{\mathrm{Tx}}^{i, j}$ of the cognitive pole $i$ on the channel $j$ is calculated as follows:

$$
\tau_{\mathrm{Tx}}^{i, j}=\frac{M_{i, j}}{C_{i, j}} \text { seconds. }
$$

Accordingly, the energy consumption during data transmission $E_{\text {data }}^{\mathrm{Tx}}$ can be calculated as follows:

$$
E_{\mathrm{data}}^{\mathrm{Tx}}=P_{\max }^{\mathrm{Tx}}\left|h_{i, j}\right| \tau_{\mathrm{Tx}}^{i, j} \text { joules. }
$$

The power consumed by the electronic circuitry of a cognitive pole during transmission mode is termed as circuit power $P^{c}$. It is a constant value and is not dependent on the rate of transmission. The amount of consumed energy due to these circuit power is given as follows:

$$
E^{\mathrm{C}}=P^{\mathrm{C}} \tau_{\mathrm{Tx}}^{i, j} \text { joules. }
$$

Eventually, the total energy consumption $E_{i, j}^{\mathrm{Tr}}$ for a cognitive pole in the transmission mode scenario can be calculated by adding (5), (6), (8), and (9) as follows:

$$
\begin{aligned}
E_{i, j}^{\mathrm{Tr}}= & \left(P^{\mathrm{Tx}} T_{\mathrm{ctr}}\right)+\left(P^{\mathrm{cs}} T_{\mathrm{cs}}^{i, j}\right)+\left(P_{\max }^{\mathrm{Tx}}\left|h_{i, j}\right| \tau_{\mathrm{Tx}}^{i, j}\right) \\
& +\left(P^{\mathrm{C}} \tau_{\mathrm{Tx}}^{i, j}\right) \text { joules. }
\end{aligned}
$$

3.2.2. Energy Consumption in the Idle Mode Scenario. Cognitive pole $i$, when not assigned a channel by the CRGW in a frame, goes into the idle state after the control message time. Energy consumption for a pole in idle mode is the sum of the energy consumed in control messaging and the energy consumed while idling. The idling duration is $T_{\text {idle }}=T-T_{\mathrm{ctr}}^{\mathrm{idle}}$. Therefore, the energy consumption by a cognitive pole when not assigned a channel by the CRGW is calculated as follows:

$$
E^{\text {idle }}=\left(P_{\max }^{\mathrm{Tx}} T_{\text {ctr }}^{\text {idle }}\right)+\left(P^{\text {idle }} T_{\text {idle }}\right) \text { joules } .
$$

Consequently, the total energy consumption in a cluster for one frame is calculated as follows:

$$
E=\sum_{\forall i, i \in \mathbb{S}} \sum_{j=1}^{N} E_{i, j}^{\mathrm{Tr}} \quad D_{i, j}+\sum_{\forall i, i \notin \mathbb{S}} E^{\mathrm{idle}}
$$

In (12), the first term represents energy consumption by the cognitive poles in transmission after they are assigned channels by the CRGW, whereas the second term depicts the poles that are not in transmission.

\section{Problem Formulation and Solution}

Energy efficiency can be defined as the throughput achieved per unit of energy consumed in a given time period, $T$. From the prescribed definition, bits per joule [29] is the metric for determining energy efficiency for the transmission line monitoring network. Dividing (4) by (12), we compute the energy efficiency of a cognitive pole network cluster as follows:

$$
\eta=\frac{R}{E} \text { bits/joule. }
$$

Subsequently, we formulate the energy efficiency maximization problem as follows:

$$
\text { Optimization problem : } \underset{\vec{d}}{\max } \eta \text {, }
$$

$$
\begin{gathered}
\text { s.t. } \sum_{j=1}^{N} D_{i, j} \leq 1, \quad i \in\{1,2, \ldots, M\}, \\
\sum_{i=1}^{M} D_{i, j} \leq 1, \quad j \in\{1,2, \ldots, N\}, \\
D_{i, j} \in\{0,1\},
\end{gathered}
$$

where $\overrightarrow{\mathbf{d}}=\left[D_{i, j}, i \in\{1, \ldots, M\}, j \in\{1, \ldots, N\}\right]$ is the assignment vector for elements $D_{i, j}$. Equation (15) confirms that each cognitive pole is assigned with a maximum of one channel only because each cognitive pole is assumed to have a single antenna. Restriction (16) ensures that only one cognitive pole is transmitting on a channel at a given time; thus, in a cluster, simultaneous transmission on a channel is prohibited. Constraint (17) indicates that $D_{i, j}$ is a binary variable. Our problem contains a nonlinear objective function and is complex in its solution.

For instance, if a cluster has $N=15$ idle channels and $M=20$ cognitive poles to transmit the data in a frame, the search space, considering all the possible assignments, is composed of $\sum_{j=0}^{N}(N ! / j !(N-j) !)(M ! /(M-j) !)$ elements. At the beginning of each frame, the CRGW solves the problem by employing the assignment scheme and broadcasts the assignment decision to all the cognitive poles. Afterward, the cognitive poles start transmission of the collected data via the assigned channels. Since the CRGW needs to make the decision at the beginning of each frame, the assignment scheme should be simple in its solution and efficient in its energy consumption. For a small number of cognitive poles and available idle channels, the optimal solution can be found by an exhaustive search; but for a generic scenario, where cognitive poles and available idle channels are larger in number, an implementable solution is sought.

We can model the channel assignment problem using weighted bipartite graphs by placing the cognitive poles in one group of vertices, $G_{1}$, and the idle channels in another group of vertices, $G_{2}$, such that $G_{1} \cap G_{2}=\phi$ (i.e., no element of a group lies in the other group) and $\eta_{i, j}$ (i.e., the energy efficiency attained when CRGW assigns a channel $j$ to pole $i$ ) is the weight of the corresponding edge. Accordingly, energy efficiency maximization corresponds to maximum weight matching. The maximum weight-matching problem can be solved using the Hungarian algorithm. Therefore, with the 


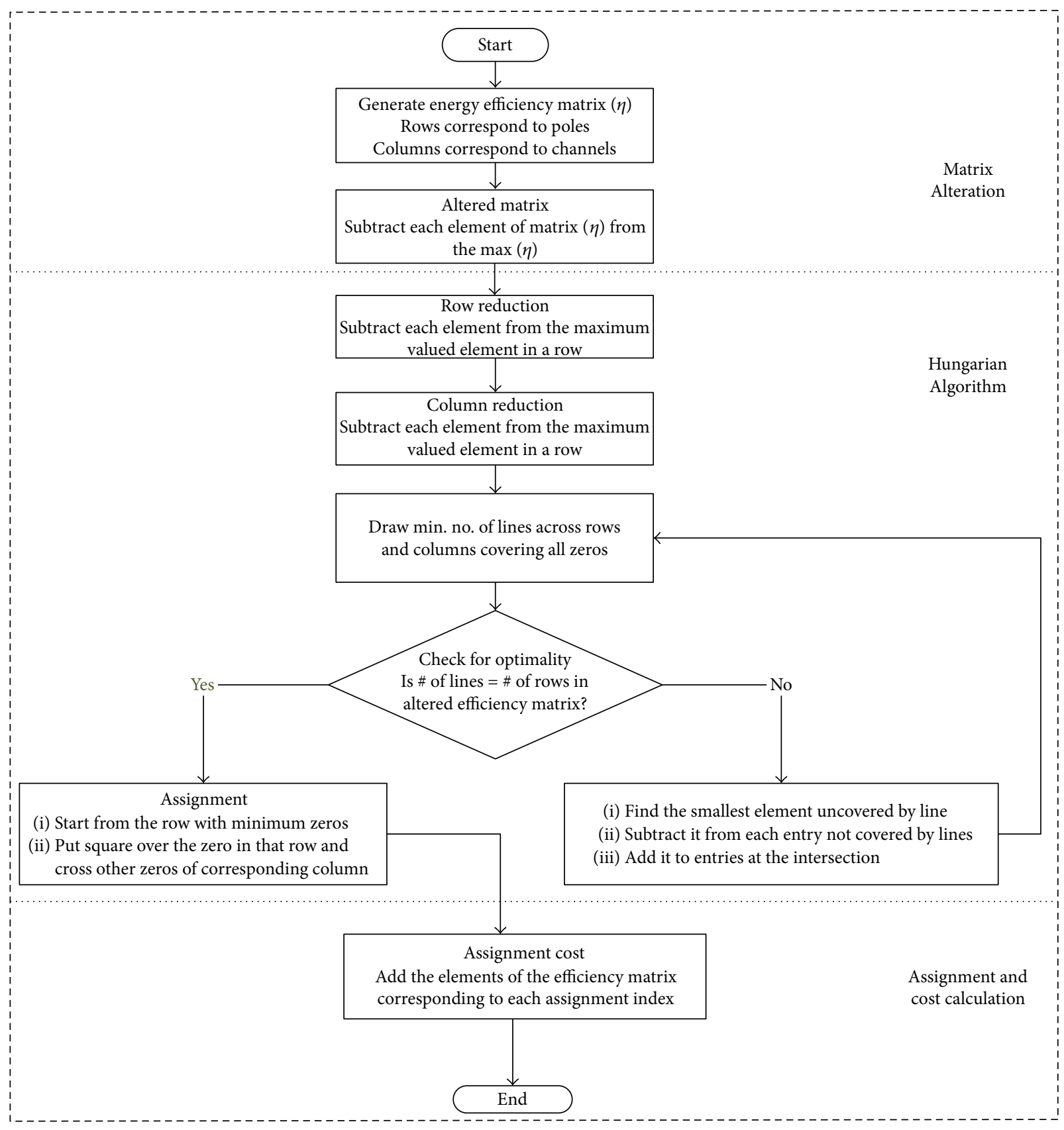

Figure 3: Flowchart of the energy-efficient channel assignment scheme (ECAS).

aim of energy efficiency maximization, we propose an energy-efficient channel assignment scheme (ECAS) based on the Hungarian algorithm to solve this problem.

The assignment procedure proposed in the ECAS is shown in Figure 3. The Hungarian method tries to minimize the objective function or the assignment cost, but our objective is to find the assignment that yields the maximum energy efficiency. For this purpose, we first alter the energy efficiency matrix to fit the setting by subtracting each element of the original efficiency matrix from the maximum valued element. Next, we apply the Hungarian algorithm to the altered efficiency matrix to retrieve the channel assignment indices for energy efficiency maximization purposes.
Stages involved in the ECAS are explained in Figure 3. Finally, we calculate the total energy efficiency value from the original matrix by adding the obtained assignment indices for each frame. The Hungarian method is a combinatorial optimization algorithm that solves the assignment problem in polynomial time. The time complexity of the Hungarian method is $O\left(|N|^{3}\right)$ [30], where $N$ is the number of available idle channels.

\section{Performance Evaluation}

We used the energy consumption, the number of available average reports, and the energy efficiency ratio as basic 
performance metrics. We presented the performance of a maximum rate heuristic assignment scheme (MRHAS) as a yardstick to compare the performance of our proposed scheme (ECAS). The MRHAS is a well-known allocation scheme, which greedily allocates the idle channel to the cognitive pole in the cluster, which attains the maximum throughput on that idle channel. The compared scheme has polynomial time complexity, that is, linear in $M$ and $N$. We also compared the results from our scheme with a random scheme (RNDM), in which idle channels are randomly assigned to the cognitive poles. For simplification of the analysis, we considered the contiguous spectrum scenario with channels of equal bandwidth for the transmission line monitoring operation. The following results were collected from 15 independent runs of the assignment for 500 consecutive frames. We set each pole to send data of 200 bits per frame when allocated a channel by the CRGW. The SNR of the channel is assumed to follow an exponential process with a mean of $3.5 \mathrm{~dB}$. The relationship between the power values is such that $P^{\mathrm{d}}<P^{\mathrm{cs}}<P_{\max }^{\mathrm{Tx}}$. The power consumption profile of a WLAN interface [31] is used in our simulation to determine the power consumption components. Transmission power consumption during the data transmission period is dependent upon the channel gain and has a maximum value $P_{\max }^{\mathrm{Tx}}$ after a channel is assigned to a cognitive pole by the CRGW. First, we assumed that poles in a cluster are fixed to $M=20$, and we studied the result of increasing the value of $N$. Next, we fixed the channels, $N=20$, and investigated the effect of increasing the poles $M$. Table 2 summarizes the symbols and parameters.

\subsection{Performance Evaluation for Energy Consumption.} Figure 4 illustrates the effect of increasing the number of channels on the energy consumption of assignment schemes for a fixed number of poles, that is, $M=20$. As it can be seen from Figure 4 that ECAS has the lowest energy consumption, whereas the reference schemes (MRHAS and RNDM) have higher energy consumption. When increasing the channels $(N)$, the energy consumption increases for a while, because more cognitive poles get the chance to transmit the observed data to the PCC. After a point (e.g., $N=20$ for ECAS), the energy consumption of the proposed scheme decreases and converges with the increasing $N$. Since there exists a large enough quantity of idle channel resources, the proposed scheme searches for more suitable channels, leading it to consume less energy. Additionally, since more channels are available, more cognitive poles can transmit collected data. Conversely, the energy consumption in the MRHAS and the RNDM scheme is not reduced significantly because they do not primarily aim to reduce energy consumption. The MRHAS scheme greedily assigns the channel which has higher energy efficiency to the subject cognitive pole without considering the overall energy efficiency for the whole cluster. Similarly, the RNDM scheme randomly chooses the channels for assignment to the subject cognitive pole consuming more energy and the energy consumption curve is nearly linear.

Figure 5 depicts the energy consumption performance of all the schemes from the effect of increasing the number of
TABLE 2: Symbols and the simulation parameters.

\begin{tabular}{lcc}
\hline Symbol & Description & Value \\
\hline$D_{i, j}$ & $\begin{array}{c}\text { Binary decision variable indicating } \\
\text { the assignment; } D_{i, j}=1 \text { if a channel } \\
\text { is assigned; else zero } \\
T\end{array}$ & $\{0,1\}$ \\
$T_{\mathrm{ctr}}^{\mathrm{Tx}}$ & $\begin{array}{c}\text { Frame duration } \\
\text { transmission scenario }\end{array}$ & $100 \mathrm{~m} / \mathrm{sec}$ \\
$T_{\mathrm{ctr}}^{\mathrm{idle}}$ & Control message duration in the & $50 \mu \mathrm{sec}$ \\
$W$ & $\quad$ idle scenario \\
$P_{\mathrm{max}}^{\mathrm{Tx}}$ & Channel bandwidth & $50 \mu \mathrm{sec}$ \\
$P^{\mathrm{cs}}$ & Maximum transmission power & $1980 \mathrm{~mW}$ \\
$P^{\mathrm{C}}$ & Channel switching power & $1000 \mathrm{~mW}$ \\
$P^{\mathrm{d}}$ & Circuity power & $210 \mathrm{~mW}$ \\
$N$ & Idling power & $990 \mathrm{~mW}$ \\
$M$ & Number of channels & {$[5: 50]$} \\
& Number of frequencies & {$[5: 50]$} \\
\hline
\end{tabular}

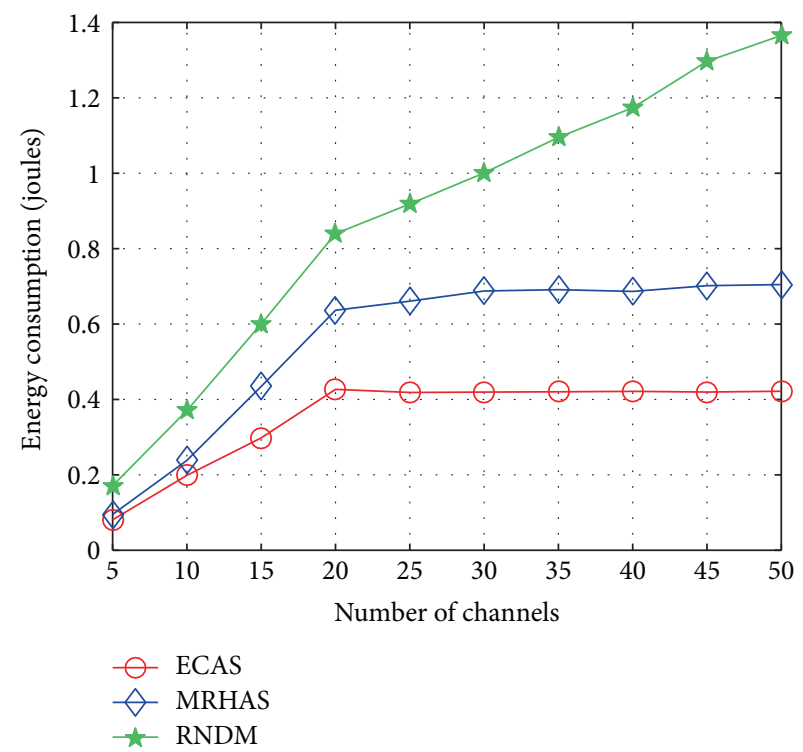

FIGURE 4: Energy consumption from increasing the number of channels.

poles to $N=20$ in a cluster. This scenario is identical to the previous one, in that an increase in the number of cognitive poles, $M$, results in an increase in energy consumption until some point (e.g., $N=20$ ). It is evident from Figure 5 that the ECAS scheme has the lowest energy consumption. The proposed scheme, ECAS, searches for the best assignment of all the cognitive poles consuming less energy overall. However, the MRHAS always attempts to allocate the best channel to the subject cognitive pole only, without considering energy efficacy in the cluster. On the other hand, RNDM scheme chooses the cognitive poles randomly for the channel assignment without taking the energy efficiency into account. 


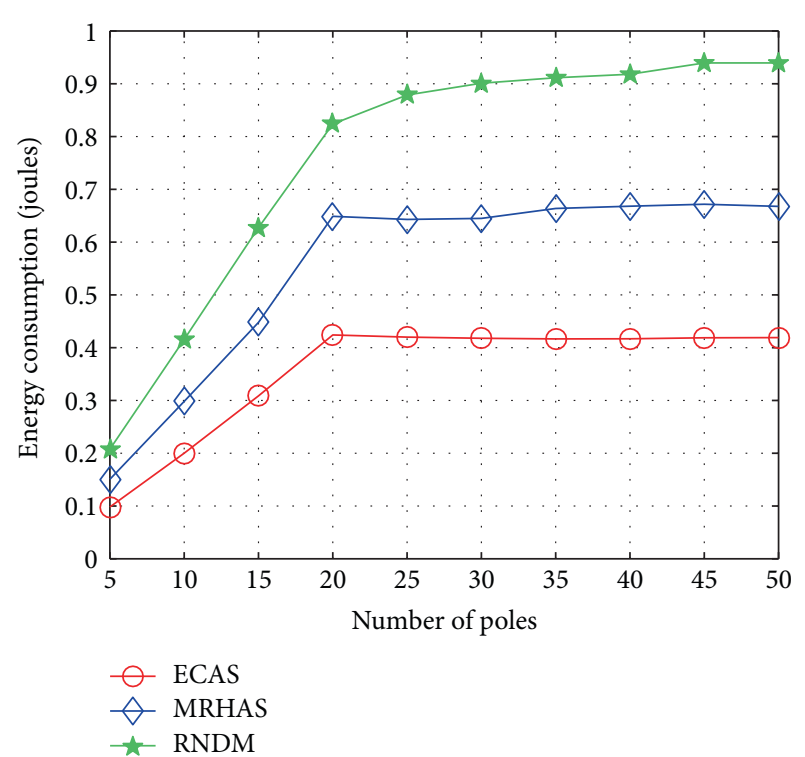

FIGURE 5: Energy consumption from increasing the number of poles.

5.2. Performance Evaluation for Average Number of Reporting. Dietrich and Dressler [32] defined the lifetime of a cluster-based sensor network as the total number of available average reports in an assignment before the battery energy of the first cognitive pole is depleted. To demonstrate the effectiveness of the proposed scheme, we assumed that all the cognitive poles in a cluster have a battery with a capacity of $15 \mathrm{mAh}$ only. We set the battery threshold level to $5 \mathrm{mAh}$ and ran scheduling for 10,000 consecutive frames. We have considered a small battery capacity for a reduction in simulation time; however, this has no effect on the performance of our results.

Figure 6 illustrates the effectiveness of the proposed scheme in total the number of available average reports when increasing channels with a fixed number of poles. It can be seen from Figure 6 that the proposed scheme, ECAS, has a huge number of available average reports before the first cognitive pole reaches the battery energy threshold level. An increase in the number of channels results in an increased number of available average reports till a certain point (e.g., $M=20$ ), and after that, the number of available average reports does not change significantly due to the reason that a fixed number of poles are participating in the transmission On the other hand, the total number of available average reports in the MRHAS decreases as the number of channels increases for a fixed number of poles (e.g., $M=20$ ) because this scheme lacks an energy efficiency perspective. Due to pure opportunistic channel assignment in MRHAS, some of the poles in the cluster may transmit on the assigned channel by consuming higher energy and deplete with energy more rapidly. Hence, this scheme consumes more energy in assignments, resulting in rapid battery energy depletion. Figure 7 depicts a scenario similar to the number of available average reports from increasing the number of poles with fixed channels (e.g., $N=20$ ).

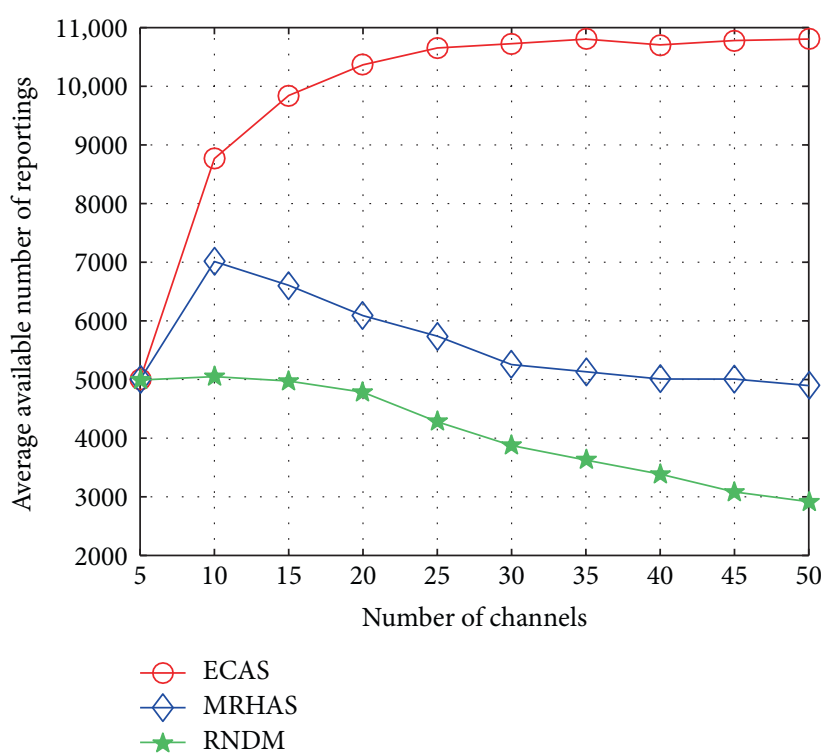

FIgURE 6: The number of available average reports with increasing number of channels.

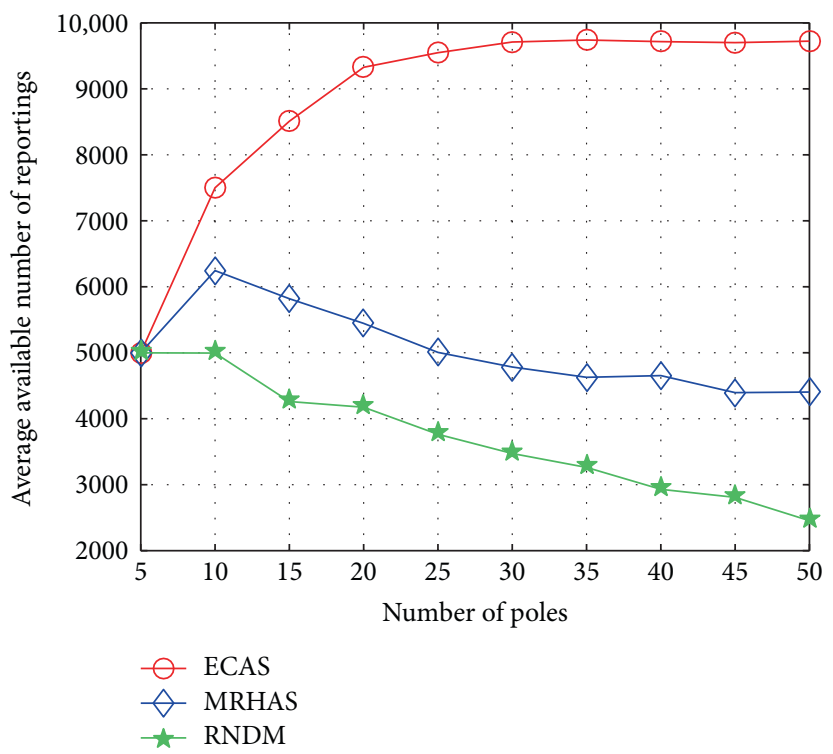

FIGURE 7: The number of available average reports from increasing the number of poles.

5.3. Performance Evaluation for the Energy Efficiency Saving Ratio. Next, we present the performance improvement in the average efficiency savings ratio (AESR) achieved in our proposed scheme over the reference schemes. We considered the pure opportunistic scheme (MRHAS) as a yardstick to calculate the energy efficiency saving ratio. The AESR is the ratio of energy consumption of the proposed scheme to the MRHAS scheme. For more clarity, we considered the ratio of the RNDM scheme to MRHAS scheme for better clarity. The AESR is calculated as follows:

$$
\mathrm{AESR}=\frac{\eta_{\mathrm{ECAS}}}{\eta_{\mathrm{MRHAS}}}
$$


Concerning AESR in Figure 8, when increasing the number of poles with fixed channels (e.g., $N=20$ ), the ECAS always has better performance than the MRHAS and the RNDM schemes, and it achieves a significant enhancement in energy efficiency for a typical operation point, $N \geq 20$, corresponding to the collected data. It is apparent that the proposed scheme can transmit more collected data with less consumption of energy. On the other hand, the MRHAS and the RNDM schemes have low performance on the energy consumption. Similarly, Figure 9 depicts the average energy efficiency improvement ratio from increasing number of channels with a fixed number of poles (e.g., $M=20$ ).

5.4. Time Delay Analysis. At the beginning of each frame, exchange of control information takes place between the cognitive poles and CRGW. The CRGW determines the transmission scheme and broadcasts it in the cluster. All these transactions are completed in control message duration which is assumed to be significantly shorter than other periods of the frame. Cognitive poles switch to the assigned channel for packet transmission.

In the proposed system model, the transmitted data packet by the cognitive pole reaches at the PCC in two stages. At the beginning of the first stage, the cognitive poles in a cluster will send the packet to the CRGW after getting channel allocation map from the CRGW in control messaging duration, which has been assumed very small. In the second stage, the CRGW will forward the data to PCC. In the following, we analyze the time delay for each stage. In the first stage, the poles in a cluster send the data packet on the assigned channel to the CRGW (cluster head). The time delay in reporting a packet by a cognitive pole to the CRGW is given as "packet size/data rate." The minimum data rate of the CR 802.22 WRAN system at upstream is $384 \mathrm{kbps}[33,34]$, and the time delay can be calculated as 0.083 seconds when the packet size is $4 \mathrm{kBytes}$. This is the time taken by one cognitive pole to transmit data to the CRGW. In the case that there are 10 cognitive poles in a cluster, the time taken by all the cognitive poles in a cluster to send data to the CRGW is $0.083 \times 10=0.836$ seconds. In the second stage, the CRGW forwards the data to the PCC. As shown in Figure 1, the CRGW is connected with the PCC on a high-speed data connection or virtual private network (VPN). Therefore, the time delay on this stage is very small and further can be neglected. The time delay of the proposed scheme is dominantly determined by the first stage. Table 3 shows time delay comparison between the linear network model and the proposed 802.22 CR WRAN-based system model.

It is obvious from the table that the time delay in the proposed scheme for reporting a message by the cognitive poles to the CRGW is significantly shorter than that of the conventional scheme [1].

\section{Conclusions}

In this work, we presented an 802.22 CR WRAN-based smart grid system for monitoring the power transmission line status that runs through terrain with hard-hitting natural and artificial threats. Efficient utilization of limited battery energy

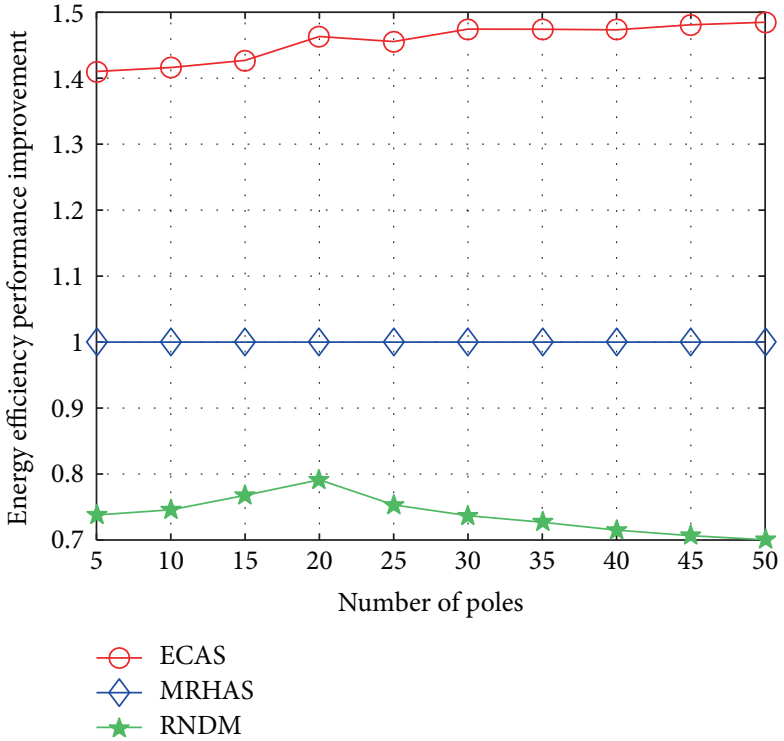

FIGURE 8: Energy efficiency performance improvement from increasing the number of poles.

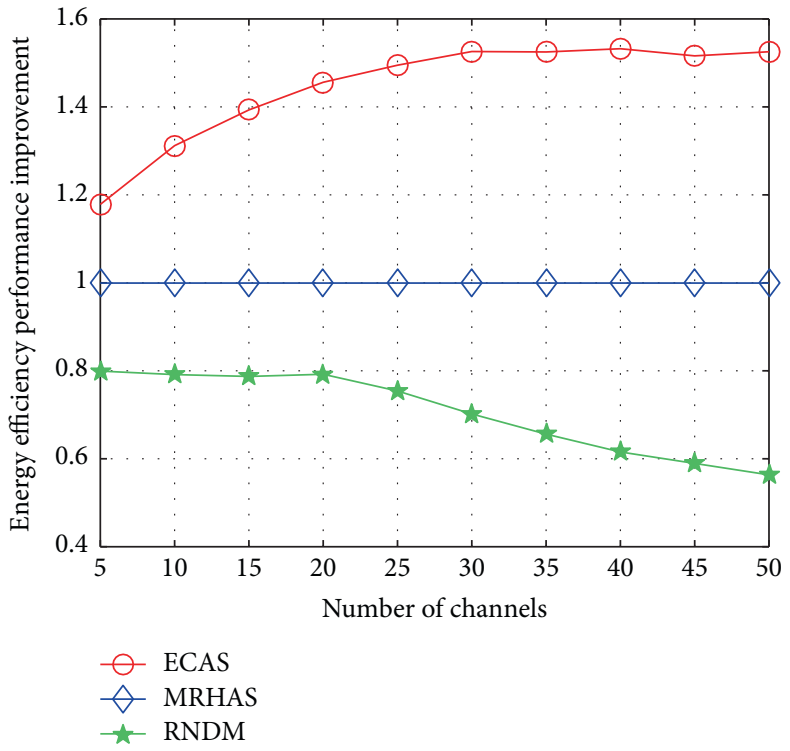

Figure 9: Energy efficiency performance improvement from increasing the number of channels.

TABLE 3: Time delay comparison between the linear and proposed 802.22 CR WRAN-based system model.

\begin{tabular}{lcc}
\hline $\begin{array}{l}\text { SCADA cycle time } \\
{[20]}\end{array}$ & $\begin{array}{c}\text { Linear network model } \\
\text { Time delay [1] }\end{array}$ & $\begin{array}{c}\text { Proposed } 802.22 \\
\text { model } \\
\text { Time delay }\end{array}$ \\
\hline $4-8$ seconds & $\begin{array}{c}162.25 \text { seconds } \approx 3 \\
\text { minutes }\end{array}$ & 0.83 seconds \\
\hline
\end{tabular}

of cognitive poles aiming the long lifetime of power line monitoring network is the design principle of this work. Considering the delicacy of battery energy, we formulated an energy-efficient channel assignment scheme (ECAS) 
based on the Hungarian algorithm running in polynomial time. We evaluated the performance of the ECAS and compared it with the maximization rate heuristic assignment scheme (MRHAS) and with a random resource assignment scheme (RNDM), as well. The proposed scheme results in high energy efficiency performance, compared to the reference schemes. Besides, throughput performance of our proposed scheme does not deteriorate under real-world operational circumstances (e.g., availability of an adequate number of idle channels). In addition, the time delay is also short as compared to a conventional scheme. Therefore, the ECAS with the objective of less energy consumption may be considered in battery-limited cognitive poles for power transmission line monitoring in the context of smart grids. For an excessive number of idle channels, all cognitive poles get the opportunity to transmit data; however, a few cognitive poles may not transmit data in a frame for a scenario where the number of available idle channels is less than the number of cognitive poles. In view of this, we plan to work on fairness in our scheme for impartial resource scheduling. Further, it will be very interesting for the future work to investigate the effects of electromagnetic field surroundings and integrate spectrum-sensing capability for an autonomous CRbased power transmission line monitoring network.

\section{Conflicts of Interest}

The authors declare that there is no conflict of interests regarding the publication of this paper.

\section{Acknowledgments}

This work was supported by the 2015 Research Fund of University of Ulsan.

\section{References}

[1] K. S. Hung, W. K. Lee, V. O. K. Li et al., "On wireless sensors communication for overhead transmission line monitoring in power delivery systems," in 2010 First IEEE International Conference on Smart Grid Communications, pp. 309-314, Gaithersburg, MD, USA, October 2010.

[2] J. Ausen, B. F. Fitzgerald, E. A. Gust, D. C. Lawry, J. P. Lazar, and R. L. Oye, "Dynamic thermal rating system relieves transmission constraint," ESMO 2006 - 2006 IEEE 11th International Conference on Transmission \& Distribution Construction, Operation and Live-Line Maintenance, 2006, Albuquerque, NM, USA, 2006.

[3] I. Ashraf, F. Boccardi, and L. Ho, "SLEEP mode techniques for small cell deployments," IEEE Communications Magazine, vol. 49, no. 8, pp. 72-79, 2011.

[4] A. A. Khan, M. H. Rehmani, and M. Reisslein, "Cognitive radio for smart grids: survey of architectures, spectrum sensing mechanisms, and networking protocols," IEEE Communications Surveys \& Tutorials, vol. 18, no. 1, pp. 860-898, 2016.

[5] J. Anatory, N. Theethayi, and R. Thottappillil, "Channel characterization for indoor power-line networks," IEEE Transactions on Power Delivery, vol. 24, no. 4, pp. 1883-1888, 2009.
[6] V. C. Gungor and F. C. Lambert, "A survey on communication networks for electric system automation," Computer Networks, vol. 50, no. 7, pp. 877-897, 2006.

[7] Y. Yang, D. Divan, R. G. Harley, and T. G. Habetler, "Design and implementation of power line sensornet for overhead transmission lines," in 2009 IEEE Power Energy Society General Meeting, pp. 1-8, Calgary, AB, Canada, July 2009.

[8] S. Malhara and V. Vittal, "Mechanical state estimation of overhead transmission lines using tilt sensors," IEEE Transactions on Power Apparatus and Systems, vol. 25, no. 3, pp. 12821290, 2010.

[9] Y. Yang, F. Lambert, and D. Divan, “A survey on technologies for implementing sensor networks for power delivery systems," in 2007 IEEE Power Engineering Society General Meeting, pp. 1-8, Tampa, FL, USA, June 2007.

[10] R. A. Len, V. Vittal, and G. Manimaran, "Application of sensor network for secure electric energy infrastructure," IEEE Transactions on Power Delivery, vol. 22, no. 2, pp. 1021-1028, 2007.

[11] J. Chen, S. Kher, and A. K. Somani, "Energy efficient model for data gathering in structured multiclustered wireless sensor network," in 2006 IEEE International Performance Computing and Communications Conference, pp. 8-388, Phoenix, AZ, USA, April 2006.

[12] J. Lin, B. Zhu, P. Zeng, W. Liang, H. Yu, and Y. Xiao, "Monitoring power transmission lines using a wireless sensor network," Wireless Communications and Mobile Computing, vol. 15, no. 14, pp. 1799-1821, 2015.

[13] Z. Wang, X. Zhao, and X. Qian, "The application and issuse of linear wireless sensor networks," in 2011 International Conference on System science, Engineering design and Manufacturing informatization, pp. 9-12, Guiyang, China, October 2011.

[14] Y. C. Wu, L. F. Cheung, K. S. Lui, and P. W. T. Pong, "Efficient communication of sensors monitoring overhead transmission lines," IEEE Transactions on Smart Grid, vol. 3, no. 3, pp. 1130-1136, 2012.

[15] B. Fateh, M. Govindarasu, and V. Ajjarapu, "Wireless network design for transmission line monitoring in smart grid," IEEE Transactions on Smart Grid, vol. 4, no. 2, pp. 1076-1086, 2013.

[16] J. I. Huertas, R. Barraza, and J. M. Echeverry, "Wireless data transmission from inside electromagnetic fields," in 2009 6th International Conference on Electrical Engineering, Computing Science and Automatic Control (CCE), pp. 1-7, Toluca, Mexico, November 2009.

[17] F. Khan, A. u. Rehman, M. Arif, M. Aftab, and B. K. Jadoon, “A survey of communication technologies for smart grid connectivity," in 2016 International Conference on Computing, Electronic and Electrical Engineering (ICE Cube), pp. 256-261, Quetta, Pakistan, April 2016.

[18] X. Lu, P. Wang, D. Niyato, D. I. Kim, and Z. Han, "Wireless charging technologies: fundamentals, standards, and network applications," IEEE Communications Surveys \& Tutorials, vol. 18, no. 2, pp. 1413-1452, 2016.

[19] F. Guo, H. Hayat, and J. Wang, "Energy harvesting devices for high voltage transmission line monitoring," in 2011 IEEE Power and Energy Society General Meeting, pp. 1-8, Detroit, MI, USA, July 2011.

[20] A. S. Cacciapuoti, M. Caleffi, F. Marino, and L. Paura, "Enabling smart grid via TV white space cognitive radio," in 2015 IEEE International Conference on Communication Workshop (ICCW), pp. 568-572, London, UK, June 2015. 
[21] O. Holland, H. Bogucka, and A. Medeisis, Opportunistic Spectrum Sharing and White Space Access: The Practical Reality, John Wiley \& Sons, Hoboken, New Jersey, 2015.

[22] M. Brew, F. Darbari, L. H. Crockett et al., "UHF white space network for rural smart grid communications," in 2011 IEEE International Conference on Smart Grid Communications (SmartGridComm), pp. 138-142, Brussels, Belgium, October 2011.

[23] B. Li, B. Zhang, J. Guo, and J. Yao, "Study on cognitive radio based wireless access communication of power line and substation monitoring system of smart grid," in 2012 International Conference on Computer Science and Service System, pp. 1146-1149, Nanjing, China, August 2012.

[24] R. Murty, R. Chandra, T. Moscibroda, and P. Bahl, "SenseLess: a database-driven white spaces network," IEEE Transactions on Mobile Computing, vol. 11, no. 2, pp. 189-203, 2012.

[25] H. Ma, L. Zheng, X. Ma, and Y. Luo, "Spectrum aware routing for multi-hop cognitive radio networks with a single transceiver," in 2008 3rd International Conference on Cognitive Radio Oriented Wireless Networks and Communications (CrownCom 2008), pp. 1-6, Singapore, Singapore, May 2008.

[26] S. Krishnamurthy, M. Thoppian, S. Venkatesan, and R. Prakash, "Control channel based MAC-layer configuration, routing and situation awareness for cognitive radio networks," in MILCOM 2005 - 2005 IEEE Military Communications Conference, Atlantic City, NJ, USA, October 2005.

[27] M. Cesana, F. Cuomo, and E. Ekici, "Routing in cognitive radio networks: challenges and solutions," Ad Hoc Networks, vol. 9, no. 3, pp. 228-248, 2011.

[28] D. Gözüpek, S. Buhari, and F. Alagöz, “A spectrum switching delay-aware scheduling algorithm for centralized cognitive radio networks," IEEE Transactions on Mobile Computing, vol. 12, no. 7, pp. 1270-1280, 2013.

[29] V. Rodoplu and T. Meng, "Bits-per-joule capacity of energylimited wireless networks," IEEE Transactions on Wireless Communications, vol. 6, no. 3, pp. 857-865, 2007.

[30] C. H. Papadimitriou and K. Steiglitz, Combinatorial Optimization: Algorithms and Complexity, Courier Corporation, Mineola, NY, USA, 1998.

[31] G. Miao, N. Himayat, Y. (Geoffrey) Li, and A. Swami, "Cross-layer optimization for energy-efficient wireless communications: a survey," Wireless Communications and Mobile Computing, vol. 9, no. 4, pp. 529-542, 2009.

[32] I. Dietrich and F. Dressler, "On the lifetime of wireless sensor networks," ACM Transactions on Sensor Networks, vol. 5, no. 1, pp. 5:1-5:39, 2009.

[33] V. Dehalwar, A. Kalam, M. L. Kolhe, and A. Zayegh, "Compliance of IEEE 802.22 WRAN for field area network in smart grid," in 2016 IEEE International Conference on Power System Technology (POWERCON), pp. 1-6, Wollongong, NSW, Australia, September-October 2016.

[34] C. W. Pyo, X. Zhang, C. Song, M. T. Zhou, and H. Harada, “A new standard activity in IEEE 802.22 wireless regional area networks: enhancement for broadband services and monitoring applications in TV whitespace," in The 15th International Symposium on Wireless Personal Multimedia Communications, pp. 108-112, Taipei, Taiwan, September 2012. 


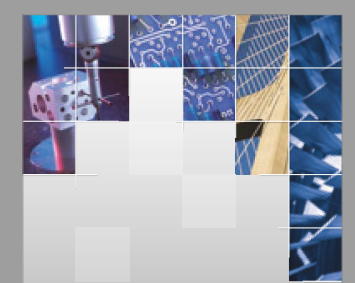

\section{Enfincering}
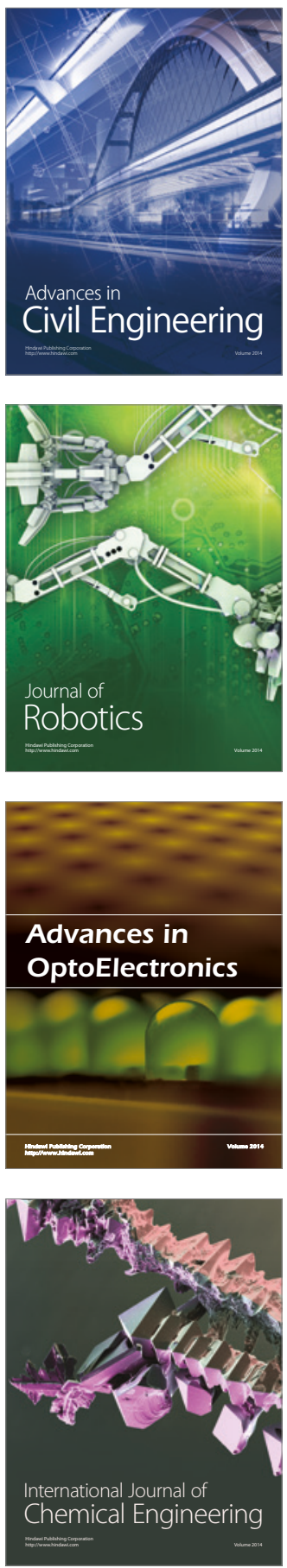

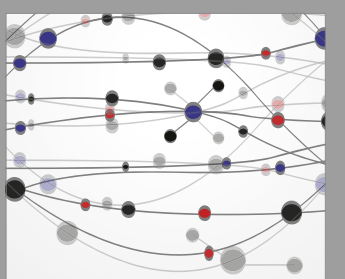

The Scientific World Journal

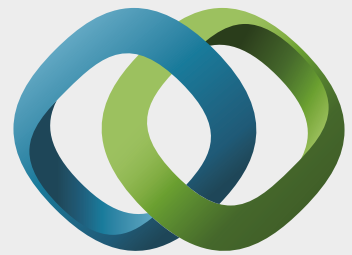

\section{Hindawi}

Submit your manuscripts at

https://www.hindawi.com
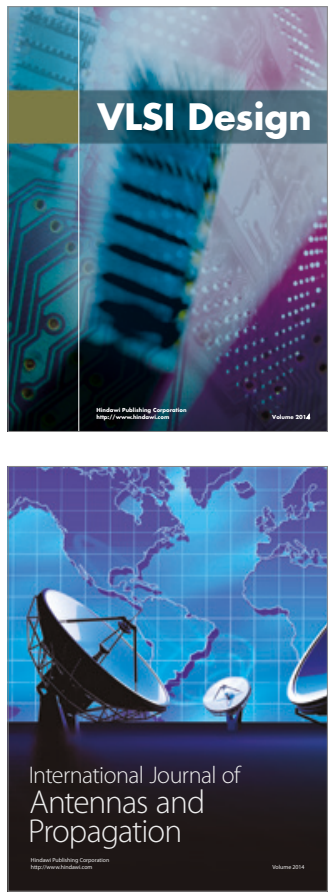

\section{Rotating}

Machinery
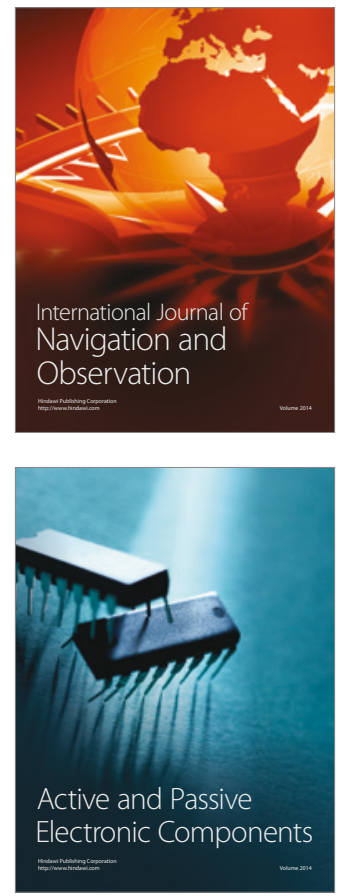
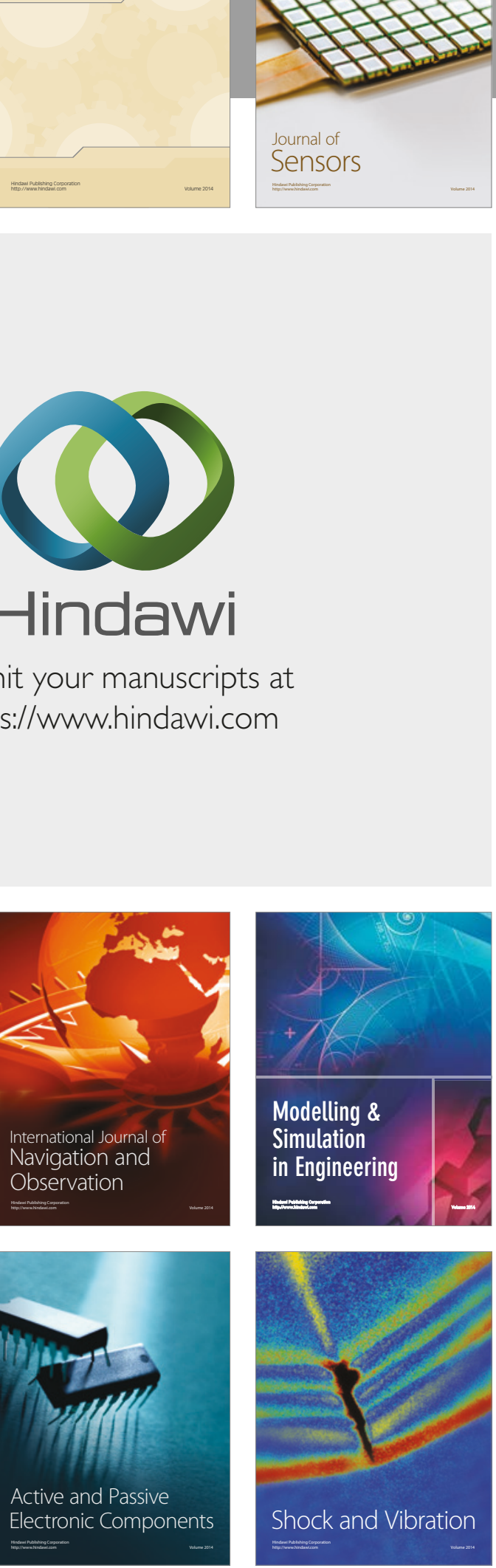
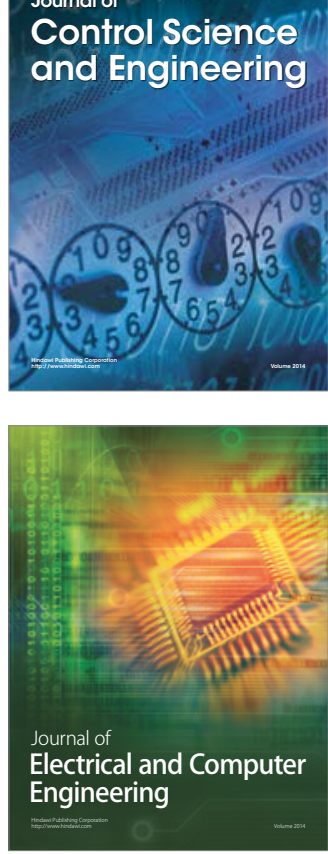

Distributed

Journal of

Control Science

and Engineering
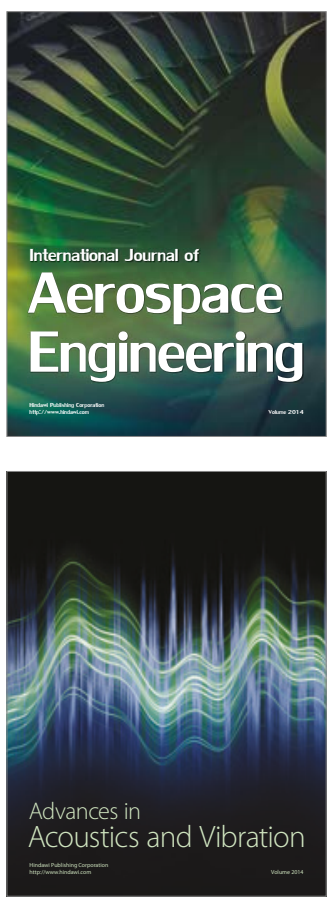

Sensor Networks 